\title{
Organisiert gegen einen profitorientierten Wohnungskonzern
}

\author{
Fünf Jahre berlinweite Vernetzung der Deutsche-Wohnen-Mieter*innen
}

Berlin gilt nicht erst seit den Großdemonstrationen der vergangenen Jahre als Hochburg der Mieter*innenbewegung. Getragen wird die Bewegung von hunderten meist lokalen Initiativen, die häufig nur wenige Monate oder Jahre bestehen. Immer wieder gibt es Versuche, diese losen Zusammenhänge in verbindlichere und übergeordnete Strukturen zu überführen. Ein Beispiel dafür ist die berlinweite Vernetzung der Deutsche-Wohnen-Mieter*innen, die seit etwa fünf Jahren besteht. Zu ihren besten Zeiten umfasste sie dutzende Delegierte von Mieter*innen-Initiativen und aktive Einzelpersonen aus allen Berliner Bezirken. Sie wehren sich gemeinsam gegen die Verwertungsstrategien des größten Vermieters Berlins. Die Deutsche Wohnen SE ist ein börsennotiertes Wohnungsunternehmen und besitzt etwa 163.00o Wohnungen, 111.00o davon in Berlin. Große Teile des Bestands waren vor seiner Privatisierung im Besitz der öffentlichen Hand. Initiiert und vorangetrieben wurde das Bündnis gegen die Aktivitäten der Deutsche Wohnen (DW) zunächst von stadtpolitisch Aktiven, die selbst nicht in deren Bestand wohnen. Ziele der Vernetzung sind es, die Aktivitäten gegen die DW zu bündeln und einen Raum für Austausch und Verabredungen zu schaffen.

Der vorliegende Text reflektiert die Organisierungserfahrungen und knüpft damit an die Strategiedebatte um Basisorganisierung an, die von Kratzsch/ Maruschke (2016) in sub/urban angestoßen wurde. Meine Rolle als Autor ist dabei nicht die eines unabhängigen Beobachters. Ich habe einige der hier beschriebenen Strukturen mit aufgebaut. Im Jahre 2016 wurde das Bündnis Otto-Suhr-Siedlung und Umgebung (BOSS\&U) von mir mitinitiiert, eine Initiative von Deutsche-Wohnen-Mieter*innen in Berlin-Kreuzberg; zudem engagiere ich mich in der AG Starthilfe der Kampagne „Deutsche Wohnen und Co. enteignen“; schließlich war ich lange Teil des Kernteams der berlinweiten Vernetzung der Deutsche-Wohnen-Mieter*innen. Im Folgenden reflektiere ich die Erfolge und Misserfolge beim Aufbau dieser Vernetzung in der Hoffnung, dass andere, die sich ähnliche Organisierungsaufgaben vornehmen, von diesen Erfahrungen profitieren können. 


\section{Meilensteine der Organisierung}

Öffentlicher Startschuss für eine berlinweite Vernetzung der Deutsche-Wohnen-Mieter*innen ist eine Aktion am 7. Juli 2016 anlässlich der Aktionärsversammlung der GSW/Deutsche Wohnen in Berlin (Kotti \& Co 2016). Bei einer Kundgebung mit mehreren Dutzend Teilnehmer*innen fordert das Bündnis der MieterInnen der Deutsche Wohnen AG den Konzern auf, „endlich wirksame Mietpreisbegrenzungen zu schaffen, die Gesetze zur ,Energetischen Modernisierung' warmmietenneutral zu gestalten und öffentlich geförderte Wohnungen zu (re-)kommunalisieren“. Die Aktion findet keine Beachtung in der Presse, die Deutsche Wohnen ist zu diesem Zeitpunkt einer breiten Öffentlichkeit nicht bekannt. Bereits im Juni 2015 hatten sich mehrere Deutsche-Wohnen-Betroffene aus Berlin abgesprochen, um gemeinsam zur Aktionärsversammlung nach Frankfurt am Main zu fahren und dort im Sinne von kritischen Aktionär*innen ein Rederecht in Anspruch zu nehmen (Kotti \& Co 2015). Beide Aktionen werden von Aktiven von Kotti \& Co angestoßen. Ein gemeinsamer E-Mail-Verteiler für DW-Betroffene aus verschiedenen Berliner Bezirken wird angelegt, der insbesondere genutzt wird, um sich zur solidarischen Begleitung von Gerichtsterminen zu verabreden.

Nachdem die berlinweite Vernetzung, eingeschlafen' war, kommt es zu einer Neubelebung im Frühjahr 2017. In dieser Zeit setzt die DW flächendeckend auf Modernisierung als Mittel zur Profitmaximierung, wodurch ein hoher Verdrängungsdruck in vielen Siedlungen entsteht. So ist es auch in der Otto-Suhr-Siedlung in Berlin-Kreuzberg. Hier gründet sich im Herbst 2016 die Mieter*inneninitiative Bündnis Otto-Suhr-Siedlung und Umgebung (BOSS\&U), um sich gemeinsam zu wehren. Diese wird von stadtpolitisch Aktiven angestoßen, insbesondere aus der Stadt AG der Interventionistischen Linken. Aus diesem Kreis heraus wird im April 2017 die berlinweite Vernetzung der Deutsche-Wohnen-Mieter*innen wiederbelebt. Gemeinsam in Aktion tritt das Bündnis am 2. Juni 2017 mit einer Kundgebung vor der Berliner Zentrale der Deutsche Wohnen, zeitgleich zur Aktionärsversammlung in Frankfurt am Main. Diese Aktion findet nun ein breites Medienecho. In der Zwischenzeit war die Presse, auch durch die Arbeit einzelner Initiativen, auf die DW und ihre Verwertungsstrategien aufmerksam geworden.

Infolge der erfolgreichen Kundgebung im Juni 2017 werden die Verwertungsstrategien der DW zum Stadtgespräch und das Bündnis wächst stetig. Bestehende Initiativen schließen sich an, neue Initiativen werden gegründet. Die Gruppe benennt sich um in „Mieter*innenprotest Deutsche Wohnen“ und gibt sich ein Logo. Ende April 2018 fällt mit einer Pressekonferenz der öffentliche Startschuss für die Kampagne „Deutsche Wohnen und Co. enteignen" (DWE), die mittels eines Volksentscheides darauf abzielt, etwa 250.00o Wohnungen profitorientierter Wohnungskonzerne wie der Deutsche Wohnen zu vergesellschaften. Beflügelt durch die erfolgreiche Auseinandersetzung mit der DW um die Karl-Marx-Allee, ist das Vernetzungstreffen im Januar 2019 mit knapp 70 Teilnehmer*innen so gut besucht wie nie. Der Kampf um die Karl-Marx-Allee wird zum Symbol des Widerstandes. Mieter*innenproteste verhindern den Verkauf von 700 Wohnungen an die Deutsche Wohnen. Statt des Konzerns kommt eine städtische Wohnungsgesellschaft durch Vorkauf zum Zuge (Kleine 2019). 
In derselben Zeit kündigt der Berliner Senat den sogenannten Mietendeckel an und reagiert damit auf den steigenden Druck der Mieter*innenbewegung. Im Zuge dessen legt die Deutsche Wohnen ihre auf Modernisierung basierende Verwertungsstrategie auf Eis. Die monatlichen Vernetzungstreffen der Protestierenden werden immer schlechter besucht, obwohl es gelingt, die Organisation auf mehr Schultern zu verteilen. Parallel dazu verlaufen sich viele lokale Initiativen. In den berlinweiten und den lokalen Strukturen bleiben wenige Einzelkämpfer*innen zurück. Allerdings engagieren sich viele davon fortan in der Enteignungskampagne und/oder in den lokalen Strukturen der Mieter*innenvereine.

\section{Berlinweite Strukturen}

Zentrales Element der Vernetzung sind monatliche, berlinweite Treffen. Hier kommen über die Jahre hinweg in der Regel zwischen 20 und 70 Personen zusammen, überwiegend DW-Mieter*innen aus allen Berliner Bezirken. Über die Zeit beteiligen sich Dutzende Personen im Alter von 20 bis 80 Jahren. Einige gründen Mieter*inneninitiativen in ihren Siedlungen und vertreten sie als Delegierte bei den Vernetzungstreffen, die in der Regel zentral in Berlin-Kreuzberg in offenen Räumen von sozialen Trägern oder Kirchengemeinden stattfinden.

Organisiert werden die Versammlungen anfangs von Aktiven wie mir, die selbst keine Deutsche-Wohnen-Mieter*innen sind. Wir kümmern uns um die Räume, die Einladung, die Tagesordnung und die Moderation. Es ist nicht leicht, Freiwillige für diese Orga-Aufgaben zu finden. Für Ungeübte es ist eine immense Herausforderung, Großgruppentreffen vorzubereiten und zu moderieren. Auch sind viele durch ihre lokalen Initiativen ohnehin schon ausgelastet. Später gelingt es, diese Aufgaben breiter zu verteilen, indem ein sechsköpfiges Kernteam gegründet wird. Hier sind nun die Aktiven, die über ihr Mietverhältnis bei der Deutsche Wohnen zur Vernetzung gestoßen sind, in der Mehrheit.

Inhaltlich dreht sich vieles um die Verwertungsstrategien der DW und den Widerstand dagegen. Breit diskutiert wird über energetische Modernisierung und mangelnde Instandsetzung sowie über überhöhte Nebenkosten oder das Thema ,smart home'. Immer wieder werden Arbeitsgruppen gebildet, um die Debatten im Plenum zu entzerren und Themen tiefergehend zu bearbeiten. Dabei arbeitet nur die AG Modernisierung langfristig auch außerhalb der monatlichen Treffen. Sie trägt viel Wissen zusammen und stellt es allen zur Verfügung. Trotz wiederholter Versuche kommt eine AG Recht nicht zustande. Zwar verabreden wir uns immer wieder zu gemeinsamen Prozessbegleitungen, aber es gelingt nicht, diese zu dokumentieren oder relevante Rechtsprechung aufzuarbeiten und juristisches Wissen verfügbar zu machen. Dabei fechten Aktive der Initiativen „Unsere Eisenbahn Siedlung“ (Barthel/Göbel 2019) oder BOSS\&U (Schneider 2018) Konflikte mit der DW vor Gericht aus, die Signalwirkung für viele andere haben. Jedoch gelingt es nicht, diese Fälle diskutierbar zu machen, geschweige denn, sie öffentlich zu begleiten. Es zeigt sich, dass ohnehin überlastete Ehrenamtliche eine derart voraussetzungsvolle Arbeit nur schwer leisten können. 
In den ersten Jahren strukturieren und moderieren jüngere Aktive die Treffen. Nicht immer gelingt es ihnen, die Älteren einzubinden, die andere Formen des Diskutierens und Sich-Organisierens gewohnt sind oder völlig ohne Vorerfahrungen zu den Treffen kommen. Weil ständig neue Leute dazukommen, ufern Vorstellungsrunden häufig aus und vieles muss immer wieder neu besprochen werden. In den ersten Jahren sind die Treffen oft überladen, ellenlange Tagesordnungen müssen in knapp vier Stunden (immer samstags von 13 bis $16 \mathrm{Uhr}$ ) abgearbeitet werden. Nie ist genug Zeit, alle Anliegen zu besprechen. Oft bleiben die Diskussionen abstrakt, verlieren sich in Details und drehen sich im Kreis. Das ehrenamtliche Kernteam ist überlastet und kann den unterschiedlichen Ansprüchen nicht gerecht werden.

Außerdem bleibt für ein wichtiges Anliegen der Vernetzung - den Austausch darüber, wie der Aufbau von Mieter*inneninitiativen vor Ort gelingt - häufig nicht genug Zeit. Als Reaktion darauf gründet sich zum einen die AG Starthilfe, eine gemeinsame Struktur mit der Kampagne DWE, die fortan den Aufbau von Mieter*inneninitiativen unterstützt[1]. Zum anderen werden die Treffen anders gestaltet. Als eine Maßnahme werden sogenannte Thementische eingeführt. Von nun an teilt sich für jeweils eine Stunde die Gruppe, und diverse Themen (Rechtliches, Modernisierung etc.) werden parallel diskutiert. Neulinge müssen an den Tisch der AG Starthilfe und bekommen eine Einführung sowie Unterstützung bei ihrem Anliegen angeboten.

Mit der Zeit entsteht eine gut gepflegte Homepage des Mieter*innenprotest Deutsche Wohnen[2]. Hier finden Interessierte Kontaktmöglichkeiten für Initiativen vor Ort und die Termine für die nächsten berlinweiten Treffen. Der E-Mail-Verteiler umfasst mittlerweile über 300 DW-Mieter*innen. Sowohl Homepage als auch Verteiler werden von wenigen Aktiven aus dem Kernteam gepflegt. Name und Logo werden am Ende der dritten Phase in ,Kampfabstimmungen“ bestimmt. Dabei setzen sich Vorschläge durch, die von den jüngeren Aktivist*innen eingebracht wurden. Viele der älteren Aktiven nehmen Logo und Namen nicht an, weil sie sich mit der Ästhetik nicht identifizieren können.

Ab Anfang 2019 sinken die Teilnehmerzahlen an den monatlichen Treffen kontinuierlich. Der Anspruch, über mehrere Jahre ein monatliches Delegiertentreffen aufrechtzuerhalten, scheint zu groß zu sein. Am Beispiel der Initiative BOSS\&U lässt sich zeigen, welche Personen überhaupt Interesse an den Vernetzungstreffen haben. BOSS\&U stößt mit der Neubelebung 2017 dazu. Aktive aus der Siedlung beteiligen sich nur im ersten Jahr und in der Regel auch nur als stille Beobachter*innen. Das ist insbesondere bemerkenswert, weil die Treffen in der Regel in der Otto-Suhr-Siedlung stattfinden. Dagegen bringen sich andere Aktive, die nicht bei der Deutsche Wohnen mieten, bis heute stark ein. Das Beispiel zeigt, dass Metastrukturen wie die Vernetzung nur von einem bestimmten Personenkreis als wichtig angesehen werden. Initiativen ohne Personen mit aktivistischem Selbstverständnis sind auf Dauer meist nicht mit Delegierten bei Vernetzungstreffen vertreten.

\section{Gemeinsame Strategien}

Es gibt von Anfang an keinen Masterplan für die Vernetzungsarbeit und auch keine einheitliche Strategie. Nach der Wiederbelebung im Frühjahr 2017 
empfiehlt das Kernteam das Erfolgsmodell der Initiative der Otto-SuhrSiedlung zur Nachahmung. Diese hatte im Zusammenspiel mit einer progressiven Bezirksregierung einen Achtungserfolg gegen die Deutsche Wohnen erzielt. Der Konzern wurde gezwungen, eine Sozialvereinbarung abzuschließen, durch die Mietererhöhungen nach Modernisierungen deutlich abgeschwächt wurden. Das Otto-Suhr-Modell ist seitdem in der Welt[3]: Bei drohender Verdrängung durch Modernisierung, das ist eine Lehre aus dieser Erfahrung, sollen sich Nachbarschaften zu einer Initiative zusammentun, um sich gemeinsam zu wehren. Die Bezirkspolitik gilt es öffentlich unter Druck zu setzen, damit sie sich mit ihren Instrumenten (,Milieuschutz') für Mieter*inneninteressen einsetzt und so die Deutsche Wohnen zu Zugeständnissen zwingen kann. Diese Strategie wurde von Initiativen in den Berliner Stadtteilen Prenzlauer Berg, Lankwitz, Neukölln, Tempelhof und Kreuzberg mit unterschiedlichem Erfolg aufgegriffen. Auf einige diese Erfahrungen wird weiter unten ausführlicher eingegangen.

Mit der Forderung nach einer Enteignung der Deutsche Wohnen durch Volksentscheid zeichnete sich ab Ende 2017 eine weitere gemeinsame Stoßrichtung ab. Anfangs ist diese Strategie allerdings hochumstritten. Initiativenvertreter*innen befürchten, dass die Enteignungsforderung an ihrer Basis als zu radikal abgelehnt werden könnte. Erst als darüber öffentlich diskutiert und sich Politiker*innen und der Mieterverein positiv darauf beziehen, wird die Forderung nach Enteignung mehrheitsfähig in der Vernetzung (Thirnthal 2019).

\section{Gemeinsame Aktivitäten}

Ein Ziel der Vernetzung ist es von Anfang an, den Deutsche-Wohnen-Mieter*innen eine Stimme zu geben. In mancher Hinsicht gelingt das auch sehr gut. Was weniger gut gelingt, ist regelmäßig Öffentlichkeitsarbeit zu machen und sich mit Pressemitteilungen oder Veranstaltungen in aktuelle stadtpolitische Debatten einzubringen. Das Vorgehen, mit einer rein ehrenamtlichen Struktur im Umlaufverfahren auf aktuelle Entwicklungen zu reagieren, erweist sich als zu langsam und kaum praktikabel.

Erfolgreich sind dagegen verschiedene Aktionen der Vernetzung. Der schlechte Ruf der Deutsche Wohnen in Berlin ist zweifelsohne darauf zurückzuführen, dass solch eine umfangreiche Vernetzung gelungen ist. Bestes Beispiel ist die Kundgebung vor der Berliner DW-Zentrale im Sommer 2017 mit knapp 200 Protestierenden. Ausnahmsweise fängt das Springer-Blatt BZ dieses Ereignis gut ein. Auf der Titelseite der Zeitung sind am folgenden Tag verschiedene DWMieter*innen aus verschiedenen Berliner Bezirken abgebildet, dieihren Ärger artikulieren (Gröning 2017). Kritisiert werden drohende Verdrängung durch Modernisierung, mangelnde Instandsetzung und überhöhte Betriebskosten. Der Artikel verschweigt freilich, dass dahinter Verwertungsstrategien des Konzerns stehen, die von den Mieter*innen durchschaut und angegriffen werden. Die Aktion hat insgesamt eine stärkende Wirkung auf die Teilnehmenden und viele, die davon erfahren. Nachdem die Deutsche Wohnen öffentlich angeprangert wurde wie nie zuvor, wachsen die Strukturen der organisierten Mieter*innen der profitorientierten Konzerne. Viele kleine und große Aktionen von Initiativen gegen Deutsche Wohnen, Vonovia, Akelius und Co. folgen. Sie 
bereiten den Weg für die Kampagne „Deutsche Wohnen und Co. enteignen“, die die Vergesellschaftung der Bestände profitorientierter Wohnungskonzerne fordert und inzwischen weltweit Beachtung findet (Vollmer 2019). Letztlich erhält so auch der Berliner Senat die Legitimation, mittels Gesetzgebung (,Mietendeckel") in das Profitmodell der Wohnungswirtschaft einzugreifen.

Während die erste Kundgebung vor der Berliner Deutsche-WohnenZentrale im Sommer 2017 für die Bewegung als Erfolg gilt, ist die zweite Kundgebung ein Jahr später am selben Ort eher ein Dämpfer, weil es nicht gelingt, den Protest auszuweiten und mehr Menschen als im Vorjahr zu mobilisieren. Hier zeigen sich zum einen die hohen Ansprüche der Aktiven und zum anderen die eingeschränkten Ressourcen. Dieses Spannungsverhältnis bleibt eine ständige Quelle der Frustration. Die eigenen Nachbar*innen zu gemeinsamen Aktionen zu motivieren, ist schwierig, noch dazu, wenn die Aktionen außerhalb des eigenen Kiezes stattfinden. Groß ist die Beteiligung in der Regel nur dann, wenn in einer Siedlung oder in einem Haus gerade ,die Hütte brennt'. Bei der nächsten Demo bleiben die Nachbar*innen dann oft wieder zu Hause.

\section{Mieter*inneninitiativen als lokale Strukturen}

Die Stärke der Vernetzung und ihrer Initiativen besteht darin, den Widerstand gegen die Deutsche Wohnen vor Ort zu organisieren. Bemerkenswert ist auch, dass sich hier Menschen außerhalb linker Zirkel und Szeneviertel zusammenfinden. Die unterschiedlichen Initiativen haben in der Regel einen Kern von Aktiven, der die Fäden zusammenhält und einen Großteil der Aufgaben übernimmt. Durch die Vernetzung werden die aktivistischen Kerne der einzelnen Initiativen identifiziert und zusammengebracht und können voneinander lernen.

In der ersten Zeit der Vernetzung gelingt es, viele bestehende Initiativen ,einzusammeln'. Damit wird ein erstes Ziel der Initiatoren erreicht. Zu nennen sind hier Gruppen aus Spandau (Bürgerinitiative an der Kappe), Kreuzberg (Kotti \& Co, BOSS\&U, Block 89), Lichtenberg (Bezirksgruppe der Berliner Mietergemeinschaft), Weißensee (Weißenseeräuber), Steglitz-Zehlendorf (MieterInnen Südwest, Mieterinitiative Südende), Treptow (Mieterprotest Baume), Prenzlauer Berg (Mieterbeirat Carl-Legien-Siedlung), Tegel (Bürgerinitiative Altes Wasserwerk - Trettach Zeile), Charlottenburg (Initiative Siedlung Westend) und Schöneberg (Initiative Schöneberger Terrassen). Ab 2017 kommt es zur Neugründungen von Initiativen, etwa in Lankwitz (Initiative Unsere Eisenbahnsiedlung), Kreuzberg (Initiative Lobeckstraße 64), Neukölln (Hufi: Hufeisensiedlung), Tempelhof (BoFuGeRi: Borussia-, Fuhrmann, Germania- und Ringbahnstraße), Charlottenburg (MIMI: Mierendorfinsel), Prenzlauer Berg (Initiative Grellstraße - Prenzlauer Allee, Initiative Toppstraße/Eberswalder Straße). Ausgelöst werden die Neugründungen insbesondere von einer Modernisierungswelle der DW. In dieser Zeit ist die Vernetzung ein Anlaufpunkt für DW-Mieter*innen, die sich zu einer Initiative zusammenschließen wollen. Mit Kotti \& Co oder den MieterInnen Südwest gibt es Vorbilder, an denen man sich orientieren kann. Die AG Starthilfe bemüht sich, den Aufbau neuer Initiativen zu unterstützen, was etwa bei der Hufi in Britz gelingt (Šustr 2019). 


\section{Schwindende Strukturen}

Ab 2019 nehmen nur noch wenige Delegierte aktiver Mieter*inneninitiativen am Vernetzungstreffen teil. Zwei Prototypen stehen für diese Entwicklung: einerseits Initiativen, die sich nicht mehr treffen, und andererseits Gruppen, die vor Ort noch aktiv sind, aber nicht mehr zu den Vernetzungstreffen kommen.

Ein typischer Fall der ersten Variante ist die BoFuGeRi-Initiative aus Tempelhof. Hier droht eine umfassende energetische Modernisierung in Nachkriegsbeständen mit (noch) niedrigen Mieten. Wie fast immer wird der Protest dagegen von wenigen aktiven Einzelpersonen, sogenannten Kernaktiven, angestoßen. Diese statten sich bei der Vernetzung mit dem nötigen Rüstzeug aus und eine erste Mieterversammlung ist mit über hundert Teilnehmer*innen ein voller Erfolg (Nowak 2018). Jedoch folgt daraus keine Verstetigung der Initiative und die Auseinandersetzung endet mit einer Sozialvereinbarung (Hartmann 2019), die zwar einige Verbesserungen gegenüber den ursprünglichen Plänen der Deutsche Wohnen mit sich bringt, jedoch von den Kernaktiven als Niederlage bewertet wird. Das Otto-SuhrModell kann hier nur teilweise adaptiert werden, weil die Bezirkspolitik in Tempelhof-Schöneberg, anders als in Friedrichshain-Kreuzberg, den Konflikt mit der Deutsche Wohnen scheut. In der Konsequenz hört die Initiative auf sich zu treffen und die verbliebene Delegierte zieht sich aus der berlinweiten Struktur zurück.

Beispiele für den zweiten Fall sind die Initiativen Lobeckstraße 64 aus Kreuzberg und Hufi aus Britz. Sie haben sich zusammengetan, um sich gegen Verdrängung durch Modernisierung zu wehren und dabei beachtliche Erfolge erzielt. Der Hufi gelingt es, ein Milieuschutzgebiet durchzusetzen und der Lobeck-Initiative, einen Baustopp zu erwirken. Durch die Vernetzung haben die beiden Gruppen wichtige Unterstützung erhalten, etwa durch den Transfer von rechtlichem, strategischem und organisatorischem Wissen. Doch nach zwei bis drei Treffen sahen die Initiativenvertreter*innen keine Notwendigkeit mehr, zu den berlinweiten Versammlungen zu kommen. Sie hatten sich geholt, was sie für ihren lokalen Kampf brauchten. Das Kernteam reagiert darauf und lädt Kernaktive beider Initiativen ein, um ihren Kampf bei einem Vernetzungstreffen als ,Fall des Monats' vorzustellen. Damit sollen den lokalen Auseinandersetzungen mehr Raum eingeräumt werden, um den anderen Teilnehmer*innen des Treffens Beispiele für erfolgreiche Kämpfe zu geben sowie die Kämpfenden durch Wissen und praktische Hilfsangebote aus der Gruppe zu unterstützen. Dieses Format kommt gut an und hat viel Potenzial.

\section{Wie weiter organisieren?}

Die Vernetzung der Deutschen-Wohnen-Mieter*innen hat über fünf Jahre einiges erreicht. Es ist uns gelungen, die Vereinzelung aufzuheben und kollektiven Widerstand gegen die Deutsche Wohnen zu ermöglichen. Es wurden viele Menschen außerhalb linker Zirkel für politisches Engagement gewonnen. Hunderte Betroffene haben sich in ihren Nachbarschaften zusammengetan, gemeinsam gekämpft und teilweise Erfolge gegen die DW erzielt. Zwar treffen sich viele lokale Initiativen nicht mehr, aber Dutzende der Kernaktiven sind an den Auseinandersetzungen gewachsen und haben voneinander gelernt. 
Inzwischen gibt es die Kampagne DWE, die von den Aktivist*innen der ersten Stunde mit viel Engagement unterstützt wird. Den Kern der Sammelstrukturen für die zweite Phase der Unterschriftensammlung für das Volksbegehren bilden sogenannte Kiezteams, die aus den anfänglichen Strukturen entstanden sind. Das Volksbegehren wird im Frühjahr 2021 beginnen, nachdem das Berliner Abgeordnetenhaus dafür im September 2020 grünes Licht gegeben hat.

Die Erfahrungen der Deutsche-Wohnen-Vernetzung zeigen, dass die Ansprüche an selbstorganisierte, ehrenamtliche Strukturen realistisch bleiben müssen. Ein monatliches, berlinweites Delegiertentreffen funktioniert nur, solange es gute Gründe gibt sich zu treffen. Eine dauerhaft hohe Aktivität vieler Mieter*innen in den Initiativen auch nach der unmittelbaren Betroffenheit ist kaum aufrechtzuerhalten. Wenn lokal die Zyklen der Auseinandersetzung zunächst abgeschlossen sind, sehen viele auch keine Notwendigkeit mehr für eine übergeordnete Struktur. Ein realistisches Ziel ist es stattdessen, die neu gewonnenen Aktiven langfristig einzubinden und als potenzielle Multiplikator*innen zu behalten, damit sie ihre Nachbar*innen wieder aktivieren können, wenn etwas ansteht. Hierfür müssen die richtigen Strukturen geschaffen werden.

Bei den Protesten hat es sich auch gezeigt, dass rein ehrenamtliche Organisierung mittelfristig ein Ressourcenproblem bekommt. Deshalb ist der These von Kratzsch/Maruschke unbedingt zuzustimmen, dass „flächendeckende und unbürokratische Basisorganisierung nicht ausschließlich in der Freizeit und als Hobby“ (2016: 110) zu leisten ist. Im Zweifel müssen auch professionelle - also bezahlte - Strukturen geschaffen werden. Allerdings dürfen ,Profis' den Ehrenamtlichen nicht die Aufgaben abnehmen. Im Gegenteil geht es darum, noch mehr Menschen zu aktivieren und dabei zu unterstützen, sich mit ihren Fähigkeiten einzubringen.

Diese Erfahrungen aufgreifend, wird es mit der Vernetzung weitergehen. Es soll wieder regelmäßige Treffen als Anlaufpunkt für Deutsche-WohnenMieter*innen geben. Bei solchen Stammtischen soll der informelle Austausch über die Probleme als DW-Mieter*in im Mittelpunkt stehen. Wer sich in seiner Nachbarschaft organisieren will, kann niedrigschwellig mit der AG Starthilfe ins Gespräch kommen und Unterstützung erhalten[4]. Eine Möglichkeit, das Ressourcenproblem zu lösen, kann in der engeren Zusammenarbeit mit den etablierten Mietervereinen bestehen. Die Stärken von professionellen Strukturen und Initiativen zu verbinden, könnte ein Schlüssel zum Erfolg für den Kampf gegen die profitorientierten Wohnungskonzerne sein.

\section{Endnote}

[1] Die AG Starthilfe gibt Wissen aus Mieter*innenprotesten an neugegründete Initiativen weiter. Mehr dazu in AG Starthilfe (2019a).

[2] http://deutsche-wohnen-protest.de (letzter Zugriff am 25.11.2020)

[3] Ausführlich zum Widerstand der Otto-Suhr-Siedlung: Strobel (2019).

[4] Informationen und Kontakt zur AG Starthilfe gibt es hier: https://www.dwenteignen. de/sich-wehren/ (letzter Zugriff am 25.11.2020). Die Gruppe hat ihr gesammeltes Erfahrungswissen in einer Broschüre zusammengetragen: AG Starthilfe (2019b). 


\section{Autor_innen}

Hannes Strobel ist Soziologe und arbeitet u. a. zum Wandel der Arbeitswelt, Digitalisierung und Gesundheit. Seit vielen Jahren engagiert er sich in der stadtpolitischen Bewegung.

hannesstrobel@gmx.de

\section{Literatur}

AG Starthilfe (2019a): Starthilfe für den Aufbau von Gegenmacht. Die AG Starthilfe gibt Wissen aus Mieter*innenprotesten an Initiativen weiter. In: Común - Magazin für stadtpolitische Interventionen, 2019/2, 12-13.

AG Starthilfe (2019b): Zusammentun! Wie wir uns gemeinsam gegen den Mietenwahnsinn wehren können. http://deutsche-wohnen-protest.de/wp-content/uploads/2019/o2/ mieterinnen_protest_deutsche_wohnen_broschuere_zusammentun_2019.pdf (letzter Zugriff am 2.10.2020).

Barthel, Ute / Göbel, Jana (2019): Miete Macht Rendite. Der ganz legale Wahnsinn in Berlin. In: RBB Fernsehen. Rundfunk Berlin-Brandenburg, 12.2.2019. https://programm.ard. $\mathrm{de} / \mathrm{TV} /$ Tipps/Tagestipps/?sendung=282051154494615 (letzter Zugriff am 25.11.2020.

Gröning, Alina-Doreen (2017): Sanierungsstau, Schimmel, hohe Betriebskosten: Diesen Mietern reichts. Protest gegen Deutsche Wohnen. In: BZ-Berlin, 2.6.2017. https:// www.bz-berlin.de/berlin/charlottenburg-wilmersdorf/sanierungsstau-schimmel-hohebetriebskosten-diesen-mietern-reichts (letzter Zugriff am 2.10.2020).

Hartmann, Philipp (2019): Bezirksamt und Deutsche Wohnen einigen sich. Modernisierung soll sozial verträglich sein. In: Berliner Woche, 7.3.2019. https://www.berliner-woche.de/ tempelhof/c-bauen/bezirksamt-und-deutsche-wohnen-einigen-sich_a203641 (letzter Zugriff am 2.10.2020).

Kleine, Jürgen (2019): Die Karl-Marx-Allee. Ein politischer Boulevard. In: Común - Magazin für stadtpolitische Interventionen, 2/2019, 19-23.

Kotti \& Co (2015): Rede auf der Hauptversammlung der Deutsche Wohnen AG. https:// kottiundco.net/2015/o6/12/rede-auf-der-hauptversammlung-der-deutsche-wohnen-ag/ (letzter Zugriff am 2.10.2020).

Kotti \& Co (2016): 7. Juli: Protest gegen Deutsche Wohnen. https://kottiundco.net/2016/ 07/03/7-juli-protest-gegen-deutsche-wohnen (letzter Zugriff am 2.10.2020).

Kratzsch, Claudia / Maruschke, Robert (2016): Basisorganisierung verändert die politische Landschaft. In: sub \urban. zeitschrift für kritische stadtforschung 2/3, 103-112.

Nowak, Peter (2018): Tempelhofer Mieter/innen organisieren sich gegen Deutsche Wohnen. In: Mieter-Echo-Online, 4.12.2018. https://www.bmgev.de/mieterecho/mieterechoonline/tempelhofer-mieterinnen-gg-deutsche-wohnen (letzter Zugriff am 2.10.2020).

Schneider, Marcel (2018): Ein Rechtsreferendar gegen Berlins Immobilienriesen. Legal Tribune Online. https://www.lto.de/recht/studium-referendariat/s/rechtsreferendarberlin-miete-modernisierung-verfahren-deutsche-wohnen-streit/ (letzter Zugriff am 2.10.2010).

Strobel, Hannes (2019): Zusammentun. Mit Milieuschutz gegen profitorientierte Wohnungsunternehmen in Berlin-Kreuzberg. In: Común - Magazin für stadtpolitische Interventionen, 2019/1, 10-11.

Šustr, Nicolas (2019): Nächster Mieterkampf Hufeisensiedlung. Aktivisten: Deutsche Wohnen plant energetische Sanierung der Wohnanlage in Britz. In: Neues Deutschland, 10.2.2019. https://www.neues-deutschland.de/artikel/1111947.deutsche-wohnenund-co-enteignen-naechster-mieterkampf-hufeisensiedlung.html (letzter Zugriff am 2.10.2020).

Tirnthal, Johanna (2019): Die Profite mit der Miete. Protest gegen hohe Mietpreise. Deutschlandfunk Kultur. 19.2.2019. In: Deutschlandfunk, 19.2.2019. https://www.deutschlandfunkkultur.de/protest-gegen-hohe-mietpreise-die-profite-mit-der-miete.976. de.html?dram:article_id=441392 (letzter Zugriff am 2.10.2020).

Vollmer, Lisa (2019): Vom Tempelhofer Feld auf die Straße. In: Zeit-Online, 25.6.2019. https://www.zeit.de/kultur/2019-06/wohnungsmarkt-wohnungspolitik-wohnungsbauberlin-baupolitik-tempelhofer-feld (letzter Zugriff am 2.10.2020). 
